\title{
Morphological and Molecular Identification of the Causal Agent of Anthracnose Disease of Avocado in Kenya
}

\author{
S. K. Kimaru $\mathbb{D}^{1},{ }^{1}$ E. Monda, ${ }^{2}$ R. C. Cheruiyot, ${ }^{1}{ }^{J}$. Mbaka, ${ }^{3}$ and A. Alakonya ${ }^{4}$ \\ ${ }^{1}$ Department of Plant Sciences, Kenyatta University, P.O. Box 43844, Nairobi, Kenya \\ ${ }^{2}$ Department of Microbiology, Kenyatta University, P.O. Box 43844, Nairobi, Kenya \\ ${ }^{3}$ Kenya Agricultural and Livestock Research Organisation, P.O. Box 220, Thika, Kenya \\ ${ }^{4}$ Jomo Kenyatta University of Agriculture and Technology, P.O. Box 62000, Nairobi, Kenya
}

Correspondence should be addressed to S. K. Kimaru; skimarul@gmail.com

Received 1 November 2017; Revised 25 January 2018; Accepted 31 January 2018; Published 27 February 2018

Academic Editor: Pierre Roques

Copyright (c) 2018 S. K. Kimaru et al. This is an open access article distributed under the Creative Commons Attribution License, which permits unrestricted use, distribution, and reproduction in any medium, provided the original work is properly cited.

\begin{abstract}
Anthracnose disease of avocado contributes to a huge loss of avocado fruits due to postharvest rot in Kenya. The causal agent of this disease has not been clear but presumed to be Colletotrichum gloeosporioides as reported in other regions where avocado is grown. The fungus mainly infects fruits causing symptoms such as small blackish spots, "pepper spots," and black spots with raised margin which coalesce as infection progresses. Due to economic losses associated with the disease and emerging information of other species of fungi as causal agents of the disease, this study was aimed at identifying causal agent(s) of the disease. A total of 80 fungal isolates were collected from diseased avocado fruits in Murang'a County, the main avocado growing region in Kenya. Fortysix isolates were morphologically identified as Colletotrichum spp. based on their cultural characteristics, mainly whitish, greyish, and creamish colour and cottony/velvety mycelia on the top side of the culture and greyish cream with concentric zonation on the reverse side. Their spores were straight with rounded end and nonseptate. Thirty-four isolates were identified as Pestalotiopsis spp. based on their cultural characteristics: whitish grey mycelium with black fruiting structure on the upper side and greyish black one on the lower side and septate spores with 3-4 septa and 2 or 3 appendages at one end. Further molecular studies using ITS indicated Colletotrichum gloeosporioides, Colletotrichum boninense, and Pestalotiopsis microspora as the causal agents of anthracnose disease in avocado. However, with this being the first report, there is a need to conduct further studies to establish whether there is coinfection or any interaction thereof.
\end{abstract}

\section{Introduction}

The anthracnose disease is a common disease with wide host range causing severe economic loss. The disease has been reported on a wide variety of crops including avocado, almond, coffee, guava, apple, dragon fruit, cassava, mango, sorghum, and strawberry causing severe economic losses [14]. The causal agents of this disease are not clear. However, species of the genus Colletotrichum and Pestalotiopsis have been reported as causal agents of anthracnose in avocado $[5,6]$. Such species includes C. gloeosporioides, C. acutatum, C. boninense, C. karstii, C. godetiae[7-11], and Pestalotiopsis versicolor [6].

Symptoms of anthracnose appear as pepper spot or speckle spot on immature fruit while still on tree and after fruit harvest during ripening as darkly, black coloured, sunken rounded spots with raised margins on fruit skins [12]. These lesions enlarge rapidly on the fruit skin and into the pulp leading to the death and rotting of the infected plant tissues [13]. The lesions may develop salmon-coloured, sticky spore masses typical of anthracnose diseases of this and many other plant species.

The avocado fruit has a high nutritional value since it contains vitamins (E, B, and C), minerals (potassium, iron, and phosphorus) and a great amount of oil [14]. In Kenya, avocado fruit is one of the most economically important fruits grown by both small and large scale farmers (HCDA, 2016). The fruit is mainly grown for fresh market but there is increasing demand from pharmaceutical, cosmetics, and vegetable oil industries (HCDA, 2016). 
Anthracnose caused by Colletotrichum gloeosporioides has been associated with severe losses of avocado fruits both in the field and after harvest as compared to Pestalotiopsis spp. whose impact is not widely studied [5,15-18]. Wasilwa et al. [19] reported that over $60 \%$ of the Kenyan avocado production cannot be marketed because of damage and low quality of fruits associated with anthracnose disease. Despite the huge losses associated with the anthracnose disease of avocado in Kenya, no cultural and molecular studies have been done to identify the causal agent(s). During the investigation, this paper aimed to identify the causal agent(s) of anthracnose of avocado in Kenya.

\section{Materials and Methods}

2.1. Fungal Isolation and Culturing. Samples of infected avocado fruits showing symptoms of anthracnose were collected from study area, Murang'a County, and brought to the laboratory for fungal isolation. The typical symptoms were small blackish spots "pepper spot" to larger blackish spots with raised margin [5]. The samples were cleaned using tap water and blotted to remove excess water. The fruits were surface sterilized using $0.5 \%$ sodium hypochlorite for 30 seconds. Small sections of the diseased area were cut aseptically and placed on hardened potato dextrose agar (PDA) in Petri dishes for fungal growth at room temperature $\left(22-25^{\circ} \mathrm{C}\right)$. The emerging fungi were subcultured to obtain pure cultures. To obtain pure cultures, single spore isolation was done as follows: cultures were flooded with sterile distilled water and conidia were scraped off the plate using sterilized wire loop and suspended in $1 \mathrm{ml}$ of sterile distilled water. A loopful of conidial suspension was spread evenly on $1.5 \%$ (wt/vol) water agar in a Petri dish and incubated at $25^{\circ} \mathrm{C}$ overnight. Using a sterilized glass needle germinated conidium was transferred onto hardened PDA in $9 \mathrm{~cm}$ diameter Petri dish and incubated at $25^{\circ} \mathrm{C}$ with a $12 \mathrm{~h}$ cycle of fluorescent light to induce growth and sporulation. To avoid bacterial contamination $0.5 \mathrm{~g} / \mathrm{l}$ of streptomycin was added to PDA at molten state of about $50^{\circ} \mathrm{C}$ [20]. Single spore pure cultures of the pathogen were preserved in the slant universal bottle and stored in the fridge at $4^{\circ} \mathrm{C}$ for later use.

The fungi, C. gloeosporioides and Pestalotiopsis spp., were morphologically identified based on cultural and microscopical characteristics using published fungal key [21, 22].

2.2. Inoculation, Mycelial Growth, and Sporulation of C. gloeosporioides Isolates. Pure cultures of C. gloeosporioides preserved in universal bottles were used. Using a sterilized surgical scalpel, sections of mycelial plugs were cut aseptically and placed on hardened PDA on $9 \mathrm{~cm}$ diameter Petri dishes and incubated for 10 days for mycelial growth.

Five-millimetre mycelia plugs from the 10-day-old pure isolates of C. gloeosporioides were aseptically cut using fivemillimetre diameter cork borer and placed individually at the centre of hardened PDA culture in $9 \mathrm{~cm}$ diameter Petri dishes. The cultures were incubated at room temperatures of ranges $22-25^{\circ} \mathrm{C}$. Mycelia diameters of the isolates were measured at days $2,4,6,8$, and 10 after inoculation. On eleventh day, the cultures were flooded with distilled water and scrapped to bring the spores into suspension. The suspension was filtered through double layer cheese cloth to remove mycelia. The spore suspension was serially diluted to $10^{-6}$ for ease of counting the spores. The spore concentration was determined by use of haemocytometer.

2.3. Conidial Morphology and Size. Using a pipette, a drop of spore suspension $\left(10^{-6}\right)$ was placed on a microscope slide, covered with a cover slip. The spores were stained with lactophenol cotton blue and observed under microscope. The shape of the spores from different isolates was noted and their sizes in terms of length and width were measured using a calibrated ocular slide and stage micrometer.

\subsection{Determination of Genetic Diversity of Fungal Isolates}

2.4.1. DNA Extraction. Pure fungal cultures in potato dextrose agar derived from a single spore from the original isolate were used. An improved fungal extraction protocol as described by Liu et al. [23] was used. About $40 \mathrm{mg}$ of mycelia was placed in an Eppendorf tube containing $2 \mathrm{ml}$ of extraction buffer (Tris- $\mathrm{HCl}, 100 \mathrm{mM}$; EDTA, $10 \mathrm{mM}$; $\mathrm{NaCl}, 1 \mathrm{M}$; SDS, $1 \%$; proteinase $\mathrm{K}, 0.05 \mathrm{mg} \mathrm{ml}^{-1}$; $\mathrm{pH} 8.0$ ) and $10 \%(\mathrm{v} / \mathrm{v})$ glass beads and ground into powder. The samples were vortexed and incubated at $65^{\circ} \mathrm{C}$ for 30 minutes. After incubation, the samples were centrifuged at $10,000 \times \mathrm{g}$ for $15 \mathrm{~min}$ and supernatant was transferred to a fresh tube. To the supernatant, $150 \mu \mathrm{l}$ of $3 \mathrm{M}$ guanidine hydrochloride was added and incubated at $-20^{\circ} \mathrm{C}$ for 10 minutes. The samples were centrifuged at $10,000 \times \mathrm{g}$ for 10 minutes. After centrifugation the supernatant was transferred to a fresh tube, and an equal volume of isopropanol was added. Samples were incubated at $-20^{\circ} \mathrm{C}$ for $3 \mathrm{~h}$. The samples were centrifuged for $10 \mathrm{~min}$ at $10,000 \times \mathrm{g}$ and thereafter $70 \%$ ethanol was added and more centrifugation done for $10 \mathrm{~min}$ at $10,000 \times \mathrm{g}$. The nucleic acid pellet obtained was air dried and dissolved in $50 \mu \mathrm{l}$ of TE buffer (Tris-HCl, $10 \mathrm{mM}, \mathrm{pH}$ 8; EDTA, $1 \mathrm{mM}$ ). The nucleic acid dissolved in TE buffer was further treated with $3 \mu \mathrm{l}$ of RNase $\left(10 \mathrm{mg} \mathrm{ml}^{-1}\right)$, to precipitate RNA at $37^{\circ} \mathrm{C}$, and the pure DNA obtained was stored at $-20^{\circ} \mathrm{C}$ for later use. The quality of DNA was determined by loading $5 \mu \mathrm{l}$ of DNA on $1 \%$ agarose before running it for 45 volts for 30 minutes and bands were noted by visualization under the UV gel imager.

\subsubsection{Polymerase Chain Reactions and Gel Electrophore-} sis. The DNA extracted from isolates of the pathogens was used as template in polymerase chain reaction. Two sets of primers were used; the first set contained the universal primers ITS1 ( $5^{\prime}$-TCCGTAGGTGAACCTGCGG$\left.3^{\prime}\right)$ and ITS4 (5' -TCCTCCGCTTATTGATATGC- $\left.3^{\prime}\right)$ targeting all fungal isolates while the other set was a primer CgInt ( $5^{\prime}$-GGGGAAGCCTCTCGCGG-3') specific to Colletotrichum gloeosporioides combined with the universal primer ITS4 (TCCTCCGCTTATTGATATGC) for the identification of the isolates. The amplified regions were subsequently sequenced to obtain sufficient information for the identification of isolates. 
TABLE 1: Cultural and morphological characteristics of the Colletotrichum gloeosporioides on PDA.

\begin{tabular}{lcccrc}
\hline \multirow{2}{*}{ Number of isolates } & \multicolumn{2}{c}{ Upper side } & Lower side & Zonation & Conidial shape \\
& Colony colour & Texture & Colour & Concentric & Cylindrical and straight \\
\hline 24 & Whitish grey & Cottony & Creamish grey & Concentric & Cylindrical and straight \\
12 & Whitish cream & Velvety & Greyish orange & Concentric & Cylindrical and straight \\
\hline
\end{tabular}

2.4.3. Agarose Gel Electrophoresis. A 1.5\% agarose gel was prepared using $1 \mathrm{x}$ TBE-buffer and stained with $5 \mu \mathrm{l}$ of SYBR Safe and poured into casting tray having a comb to solidify. The first well of the solid gel was loaded with $1.5 \mu \mathrm{l} 1 \mathrm{~Kb}$ marker, followed by $2 \mu \mathrm{l}$ of each amplified product and a control at the end. The gel was connected to electric voltage of 100 volts for 45 minutes to allow migration of amplified PCR products. The DNA bands formed were visualized under UV light and images photographed using a camera connected to a computer.

2.4.4. DNA Cleaning and Sequencing. The amplified products of the target fragments obtained above were cleaned using the Qiagen PCR cleaning kit according to the manufacturer instructions. The cleaned fragments were submitted for Sanger sequencing at Inqaba Africa Genomic platform in South Africa together with the primers used for amplification (ITS1 and ITS4 and ITS4 and CgInt).

2.5. Bioinformatics Analysis. The sequences obtained from Inqaba Africa Genomic platform at South Africa were trimmed before subjecting them to alignment with other Genbank sequences. Gene alignment was done using BioEdit software version while phylogenetic analysis was done by Mega Molecular Evolutionary Genetic Analysis version 7.1.8.

Alignment of sequences of the isolates and phylogenetic analyses was done using Mega 7.18 [24]. The maximum likelihood trees were obtained using the Close-NeighbourInterchange algorithm while missing data and gaps were eliminated [25]. Clade stability of the resultant phylogenetic tree was based on bootstrap analysis with 1000 replicates [26]. Evolutionary distances in form of number of base substitution per site were computed using the Maximum Composite Likelihood method [24].

\section{Results}

3.1. Fungal Isolates. A total of 80 fungal isolates from diseased avocado fruits showing symptoms of anthracnose (Figure 1) collected from the study area were obtained. The isolates were identified based on cultural morphological characteristic on PDA and spore characteristics as observed under microscope $[27,28]$.

A total of 46 isolates had whitish, greyish, or creamish colour and cottony, velvety mycelium on the top side and greyish cream with circular orange-pinkish colour on the reverse side, Figure 2(a). Their spores were straight with rounded end (Figure 2(b)), typical of C. gloeosporioides [29]. The remaining 34 isolates had whitish grey mycelium with black fruiting structure on the upper side and greyish black

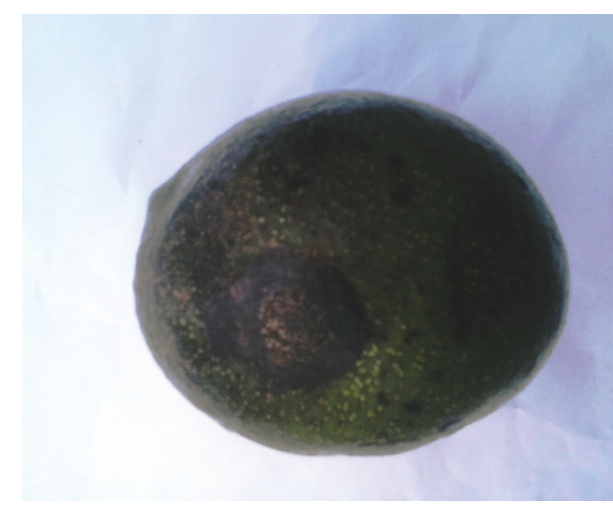

FIGURE 1: Anthracnose symptoms on avocado fruit.

one on the lower side (Figure 2(c)). Their spores had 3-4 septa and 2 or 3 appendages at one end characteristics of Pestalotiopsis microspora [30] (Figure 2(d)).

The isolates were further confirmed through Koch's postulate where ten-day-old pure cultures of the isolates growing on PDA were used to inoculate healthy ripe avocado fruit, Fuerte variety. After two days, a characteristic black spot was formed by both Colletotrichum and Pestalotiopsis isolates. Each of the reisolated fungi from the diseased fruit showed similar morphological, cultural, and spore characteristics as initial isolates.

The Colletotrichum isolates were subjected to more detailed study while the Pestalotiopsis spp. was identified further at molecular level using universal primers ITS1 and ITS4.

3.2. The Mycelial Growth of Colletotrichum Isolates. The Colletotrichum gloeosporioides isolates from the study area grew rapidly on the PDA medium covering the whole surface of the Petri dish in 10-12 days after inoculation. The mycelial colour of the isolates varied between whitish grey, whitish cream, and greyish pink on the upper side of the culture (Table 1). Similarly, the lower side of the cultures had creamish grey, greyish orange, and grey (Table 1). In terms of mycelia structure, cottony one was observed in 24 isolates as compared to velvety one observed in 22 isolates (Table 1 ). There was significant difference $\left(\chi^{2}=23.455, \mathrm{df}=2\right.$, and $P<0.001)$ among the observed and expected frequencies for the cultural texture and colour of various Colletotrichum gloeosporioides isolates (Table 1).

3.3. Mycelial Growth of Colletotrichum gloeosporioides Isolates. The mycelial diameter of the isolates showed significant 


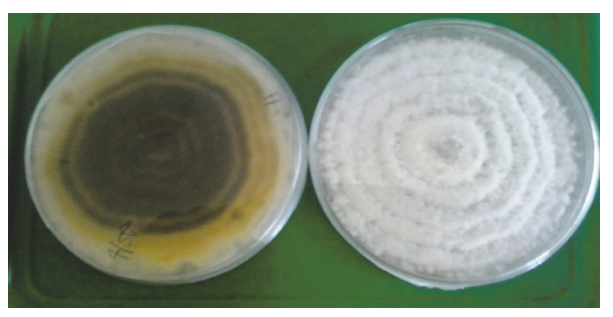

(a)

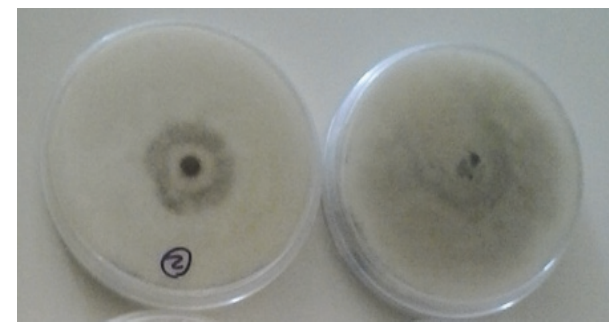

(c)

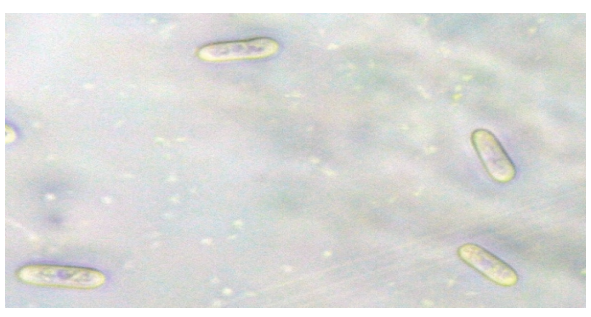

(b)

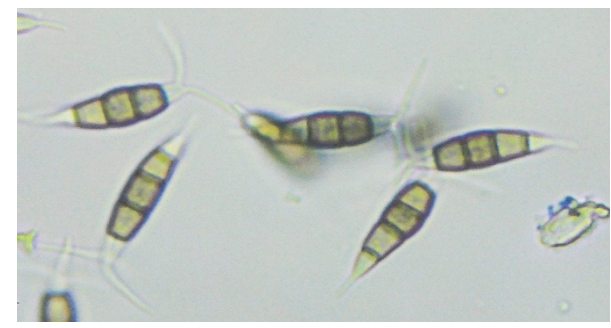

(d)

Figure 2: Mycelia of Colletotrichum gloeosporioides (a) and Colletotrichum gloeosporioides spores (b) ( $\times 400)$ and mycelia of Pestalotiopsis microspora (c) and Pestalotiopsis microspora spores $(\mathrm{d})(\times 400)$.

differences $(P<0.05)$ throughout the growth period (Table 2). However, they exhibited similar trend in growth with day two having the least and day 10 having the largest diameter per isolate (Table 2). The radial diameter of all the isolates ranged from 0.3 to $0.93 \mathrm{~cm}$ and 2.37 to $4.5 \mathrm{~cm}$ in day 2 and day 10 , respectively (Table 2 ).

3.4. Sporulation of the Colletotrichum Isolates. Sporulation of Colletotrichum gloeosporioides isolates exhibited a wide range of mean number of spores per isolate ranging from the lowest $0.65 \times 10^{6}$ to the highest $9.00 \times 10^{6}$ spores per $\mathrm{ml}$ (Table 2). These mean number of spores per $\mathrm{ml}$ differed significantly at $P<0.05$ among isolates. Isolate s1 had the highest mean number of $9.0 \times 10^{6}$ per $\mathrm{ml}$ which was significantly different from the rest at $P<0.05$ (Table 2). A total of 24 isolates had mean number of spores lower than the tabulated mean of all the isolates of $3.49 \times 10^{6}$ per isolate.

3.5. Conidial Morphology and Size. All the spores observed were cylindrical and straight with smooth round end. The spore size varied significantly at $P<0.05$ among isolates ranging from 3.0 to $5.0 \mu \mathrm{m}$ in width and 10.3 to $18.2 \mu \mathrm{m}$ in length (Table 3). The spore widths of isolates s1, s40, and s44 were highest at $5.0 \mu \mathrm{m}$ and they differed significantly from the rest at $P<0.05$ (Table 3). Similarly, these isolates produced the longest spores of $18.2,18.0$, and $18.0 \mu \mathrm{m}$ for isolate $\mathrm{s} 1, \mathrm{~s} 40$, and $\mathrm{s} 44$, respectively. Isolates s 37 and $\mathrm{s} 46$, however, produced the smallest spores having a mean of $3.0 \mu \mathrm{m}$ in width and $10.3 \mu \mathrm{m}$ in length. Thirty-one isolates produced spores having width within the range of $3.1-3.5 \mu \mathrm{m}$ which did not differ significantly at $P \geq 0.05$ (Table 3 ). Overall, spore size in terms of width and length differed significantly at $P<0.05$ among isolates.
3.6. Phylogenetic Studies of Colletotrichum gloeosporioides and Pestalotiopsis microspora. The molecular identification of C. gloeosporioides and Pestalotiopsis spp. was inferred from 13 sequences of $C$. gloeosporioides and 10 sequences of Pestalotiopsis isolates. A phylogenetic tree (Figure 1) was made of sequences from C. gloeosporioides, Pestalotiopsis microspora, and reference sequences from the Genbank (Figure 3). The identity of the isolates of both Colletotrichum and Pestalotiopsis to the Genbank isolates ranged from 98\% to $100 \%$.

The phylogenetic analysis of the isolates and references from the Genbank resulted into three clades: Clade 1 (Colletotrichum gloeosporioides), Clade 2 (Colletotrichum boninense), and Clade 3 (Pestalotiopsis microspora) (Table 4 and Figure 3).

\section{Discussion and Conclusion}

4.1. Cultural and Morphological Characteristics of Colletotrichum gloeosporioides and Pestalotiopsis microspora Isolates. The fungal isolates from diseased avocado fruits showing symptoms of anthracnose collected from the study area varied significantly in their cultural characteristics on PDA media in terms of texture and colour. A total of 46 isolates had whitish, greyish, or creamish colour and cottony, velvety mycelium on the top side and greyish cream with circular orange-pinkish colour on the reverse side. Similar cultural characteristics among Colletotrichum gloeosporioides isolates from avocado fruits were observed by [31]. Further, Chowdappa et al. [32] also noted the wide cultural variations among $C$. gloeosporioides isolates. The mycelial growth, however, had uniform radial growth characterised by circular ring-like patterns common to C. gloeosporioides. Sharma and Kulshrestha [4] reported similar mycelial growth 


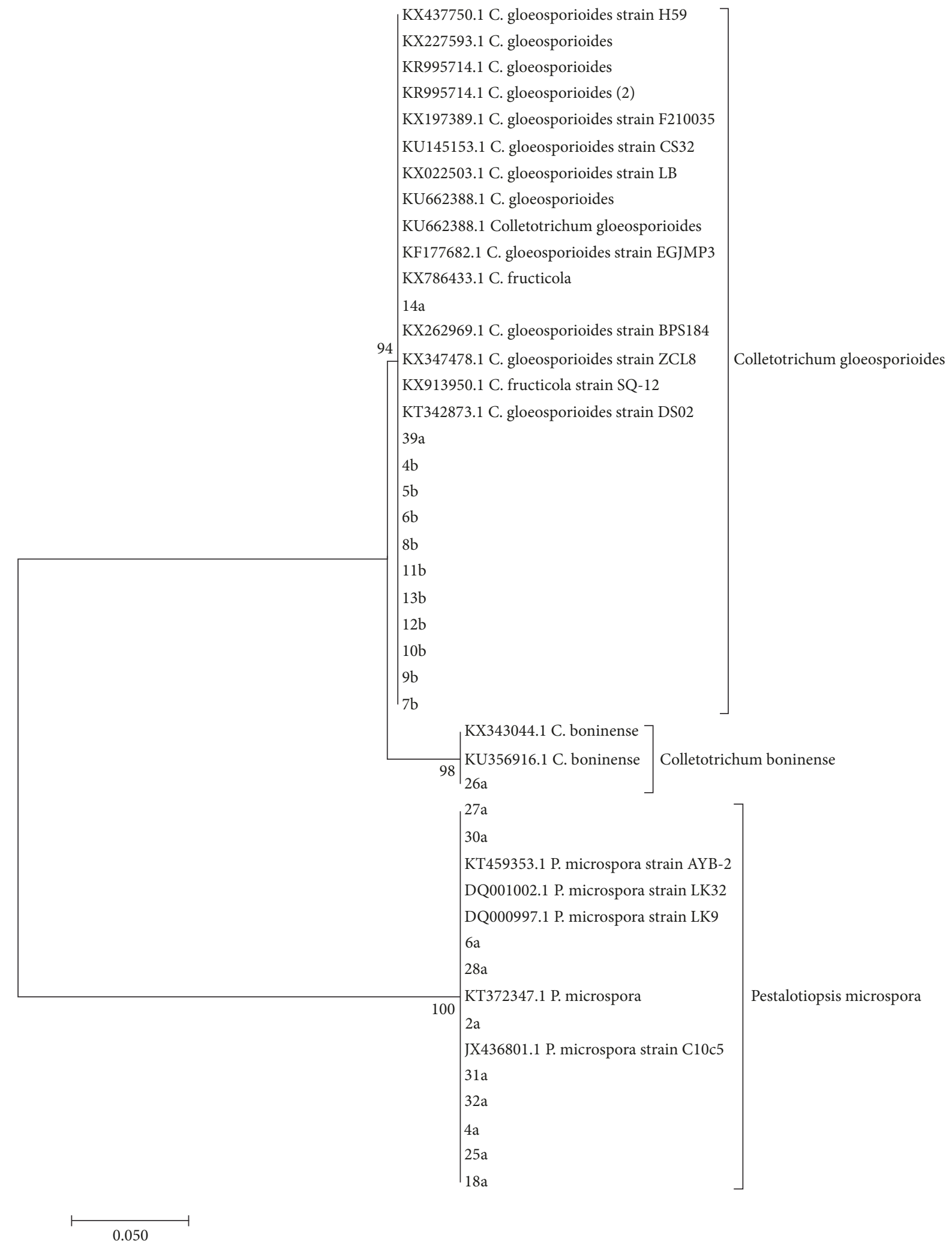

FIGURE 3: Maximum likelihood tree showing the relationship of Colletotrichum gloeosporioides complex and Pestalotiopsis microspora isolates based on ITS region. 
TABLE 2: Daily mycelial growth and mean number of spores of Colletotrichum gloeosporioides isolates.

\begin{tabular}{|c|c|c|c|c|c|c|}
\hline Isolate & Day 2 & Day 4 & Day 6 & Day 8 & Day 10 & Mean number of spores $\left(10^{6} \mathrm{ml}\right)$ \\
\hline s1 & $0.77^{* \mathrm{bcd}}$ & $1.10^{\mathrm{jklmn}}$ & $1.33^{\mathrm{pq}}$ & $2.57^{1}$ & $3.57^{\mathrm{m}}$ & $9.00^{* \mathrm{a}}$ \\
\hline s40 & $0.63^{\text {defgh }}$ & $1.02^{\mathrm{mno}}$ & $1.60^{\text {no }}$ & $3.05^{\mathrm{ijk}}$ & $4.00^{\text {efghij }}$ & $6.00^{\mathrm{b}}$ \\
\hline s44 & $0.57^{\mathrm{fgh}}$ & $1.67^{\mathrm{d}}$ & $2.23^{\mathrm{def}}$ & $3.77^{\mathrm{ab}}$ & $4.03^{\text {efghi }}$ & $5.67^{\mathrm{bc}}$ \\
\hline s3 & $0.63^{\text {defgh }}$ & $1.02^{\mathrm{mno}}$ & $1.60^{\text {no }}$ & $3.05^{\mathrm{ijk}}$ & $4.00^{\text {efghij }}$ & $5.33^{\mathrm{bc}}$ \\
\hline s5 & $0.57^{\mathrm{fgh}}$ & $1.67^{\mathrm{d}}$ & $2.23^{\mathrm{def}}$ & $3.77^{\mathrm{ab}}$ & $4.03^{\text {efghi }}$ & $5.00^{\mathrm{bc}}$ \\
\hline s6 & $0.65^{\text {cdefgh }}$ & $1.43^{\text {efg }}$ & $2.12^{\mathrm{efg}}$ & $3.45^{\text {bcdefgh }}$ & $4.23^{\mathrm{bcde}}$ & $5.00^{\mathrm{bcd}}$ \\
\hline s19 & $0.63^{\text {defgh }}$ & $1.47^{\mathrm{ef}}$ & $2.33^{\mathrm{de}}$ & $3.77^{\mathrm{ab}}$ & $4.17^{\mathrm{defg}}$ & $5.00^{\mathrm{bcd}}$ \\
\hline$s 21$ & $0.53^{\text {gh }}$ & $1.03^{\operatorname{lmno}}$ & $1.73^{\operatorname{lmn}}$ & $3.30^{\text {defghij }}$ & $3.90^{\mathrm{hijkl}}$ & $5.00^{\mathrm{bcd}}$ \\
\hline s10 & $0.50^{\mathrm{h}}$ & $1.17^{\mathrm{ijk} l m}$ & $1.83^{\mathrm{ijk} k \mathrm{~m}}$ & $3.23^{\text {fghij }}$ & $3.80^{\mathrm{ijk} k \mathrm{~m}}$ & $5.00^{\mathrm{bcd}}$ \\
\hline s18 & $0.63^{\text {defgh }}$ & $1.10^{\mathrm{jklmn}}$ & $1.97^{\text {ghijk }}$ & $3.40^{\text {cdefgh }}$ & $4.07^{\mathrm{efgh}}$ & $4.67^{\text {bcde }}$ \\
\hline s25 & $0.63^{\text {defgh }}$ & $1.10^{\mathrm{jklmn}}$ & $1.97^{\text {ghijk }}$ & $3.40^{\text {cdefgh }}$ & $4.07^{\mathrm{efgh}}$ & $4.67^{\text {bcde }}$ \\
\hline s 28 & $0.60^{\text {efgh }}$ & $0.93^{\text {no }}$ & $1.20^{\mathrm{q}}$ & $1.47^{\mathrm{n}}$ & $2.60^{\mathrm{n}}$ & $4.67^{\text {bcde }}$ \\
\hline s14 & $0.60^{\text {efgh }}$ & $0.93^{\text {no }}$ & $1.20^{\mathrm{q}}$ & $1.47^{\mathrm{n}}$ & $2.60^{\mathrm{n}}$ & $4.33^{\text {bcde }}$ \\
\hline s17 & $0.60^{\mathrm{efgh}}$ & $0.87^{\circ}$ & $1.73^{\operatorname{lmn}}$ & $3.63^{\mathrm{abcd}}$ & $4.17^{\operatorname{defg}}$ & $4.33^{\text {bcde }}$ \\
\hline s2 & $0.60^{\text {efgh }}$ & $0.87^{\circ}$ & $1.73^{\operatorname{lmn}}$ & $3.63^{\mathrm{abcd}}$ & $4.17^{\mathrm{defg}}$ & $4.00^{\text {cdef }}$ \\
\hline $\mathrm{s} 22$ & $0.50^{\mathrm{h}}$ & $1.43^{\text {efg }}$ & $2.03^{\text {fghi }}$ & $3.67^{\mathrm{abc}}$ & $4.07^{\mathrm{efgh}}$ & $4.00^{\text {cdef }}$ \\
\hline s 24 & $0.70^{\text {cdef }}$ & $1.27^{\text {ghij }}$ & $1.97^{\text {ghijk }}$ & $3.63^{\mathrm{abcd}}$ & $4.10^{\mathrm{efgh}}$ & $4.00^{\text {cdef }}$ \\
\hline $\mathrm{s} 29$ & $0.60^{\text {efgh }}$ & $1.20^{\mathrm{hijkl}}$ & $1.70^{\mathrm{lmno}}$ & $3.70^{\mathrm{abc}}$ & $4.00^{\text {efghij }}$ & $3.67^{\mathrm{def}}$ \\
\hline s30 & $0.73^{\text {bcde }}$ & $1.37^{\mathrm{fgh}}$ & $2.03^{\text {fghi }}$ & $3.60^{\text {abcde }}$ & $4.20^{\text {cdef }}$ & $3.33^{\mathrm{defg}}$ \\
\hline s43 & $0.87^{\mathrm{ab}}$ & $1.57^{\mathrm{de}}$ & $2.80^{\mathrm{b}}$ & $3.70^{\mathrm{abc}}$ & $4.03^{\text {efghi }}$ & $3.33^{\operatorname{defg}}$ \\
\hline s13 & $0.60^{\text {efgh }}$ & $1.10^{\mathrm{jklmn}}$ & $1.65^{\mathrm{mno}}$ & $2.82^{\mathrm{kl}}$ & $4.37^{\mathrm{abcd}}$ & $3.33^{\operatorname{defg}}$ \\
\hline s15 & $0.60^{\text {efgh }}$ & $1.00^{\mathrm{mno}}$ & $1.60^{\mathrm{no}}$ & $2.97^{\mathrm{jk}}$ & $4.03^{\text {efghi }}$ & $3.17^{\mathrm{efg}}$ \\
\hline s4 & $0.67^{\text {cdefg }}$ & $1.20^{\mathrm{hijkl}}$ & $1.97^{\text {ghijk }}$ & $3.43^{\text {bcdefgh }}$ & $4.13^{\text {Defgh }}$ & $3.00^{\text {efg }}$ \\
\hline s8 & $0.93^{\mathrm{a}}$ & $1.90^{c}$ & $3.23^{\mathrm{a}}$ & $3.57^{\text {bcdef }}$ & $4.10^{\mathrm{Efgh}}$ & $3.00^{\text {efg }}$ \\
\hline s38 & $0.50^{\mathrm{h}}$ & $1.43^{\text {efg }}$ & $2.03^{\text {fghi }}$ & $3.30^{\text {defghij }}$ & $4.50^{\mathrm{a}}$ & $3.00^{\text {efg }}$ \\
\hline s16 & $0.63^{\text {defgh }}$ & $2.18^{\mathrm{b}}$ & $2.37^{\mathrm{cd}}$ & $3.28^{\text {efghij }}$ & $3.65^{\mathrm{Lm}}$ & $3.00^{\text {efg }}$ \\
\hline$s 26$ & $0.80^{\mathrm{abc}}$ & $1.13^{\mathrm{jklm}}$ & $1.88^{\mathrm{hijkl}}$ & $3.18^{\text {ghij }}$ & $3.90^{\mathrm{Hijkl}}$ & $3.00^{\text {efg }}$ \\
\hline s 45 & $0.80^{\mathrm{abc}}$ & $1.90^{c}$ & $2.58^{\mathrm{bc}}$ & $3.15^{\mathrm{hijk}}$ & $3.93^{\text {Ghijk }}$ & $3.00^{\text {efg }}$ \\
\hline s9 & $0.67^{\text {cdefg }}$ & $1.32^{\text {fghi }}$ & $2.08^{\mathrm{fgh}}$ & $3.43^{\text {bcdefgh }}$ & $4.02^{\text {Efghij }}$ & $2.67^{\mathrm{fg}}$ \\
\hline $\mathrm{s} 12$ & $0.70^{\text {cdef }}$ & $1.37^{\mathrm{fgh}}$ & $2.10^{\mathrm{fgh}}$ & $3.37^{\text {cdefghi }}$ & $4.43^{\mathrm{Abc}}$ & $2.67^{\mathrm{fg}}$ \\
\hline s 20 & $0.77^{\mathrm{bcd}}$ & $1.23^{\mathrm{hijk}}$ & $1.60^{\text {no }}$ & $3.13^{\text {hijk }}$ & $3.97^{\text {Fghij }}$ & $2.33^{\text {fgh }}$ \\
\hline s27 & $0.80^{\mathrm{abc}}$ & $2.63^{\mathrm{a}}$ & $3.40^{\mathrm{a}}$ & $3.93^{\mathrm{a}}$ & $4.13^{\text {Defgh }}$ & $2.33^{\text {fgh }}$ \\
\hline s31 & $0.60^{\text {efgh }}$ & $1.03^{\operatorname{lmno}}$ & $1.50^{\mathrm{op}}$ & $2.50^{1}$ & $3.77^{\mathrm{Jklm}}$ & $2.33^{\mathrm{fgh}}$ \\
\hline s32 & $0.57^{\text {fgh }}$ & $0.93^{\text {no }}$ & $1.33^{\mathrm{pq}}$ & $2.03^{\mathrm{m}}$ & $2.37^{\mathrm{n}}$ & $2.33^{\mathrm{fgh}}$ \\
\hline s33 & $0.30^{\mathrm{i}}$ & $0.40^{\mathrm{p}}$ & $0.97^{\mathrm{r}}$ & $1.43^{\mathrm{n}}$ & $2.60^{\mathrm{n}}$ & $2.33^{\text {fgh }}$ \\
\hline s35 & $0.70^{\text {cdef }}$ & $1.03^{\mathrm{lmno}}$ & $1.70^{\operatorname{lmno}}$ & $3.25^{\text {fghij }}$ & $4.07^{\mathrm{Efgh}}$ & $2.00^{\mathrm{gh}}$ \\
\hline s36 & $0.70^{\text {cdef }}$ & $1.17^{\mathrm{ijk} k \mathrm{~m}}$ & $1.97^{\text {ghijk }}$ & $3.63^{\mathrm{abcd}}$ & $4.10^{\mathrm{Efgh}}$ & $2.00^{\mathrm{gh}}$ \\
\hline s41 & $0.63^{\text {defgh }}$ & $1.10^{\mathrm{jklmn}}$ & $1.77^{\mathrm{klmn}}$ & $2.83^{\mathrm{kl}}$ & $4.00^{\text {Efghij }}$ & $2.00^{\mathrm{gh}}$ \\
\hline s7 & $0.60^{\text {efgh }}$ & $1.13^{\mathrm{j} \mathrm{klm}}$ & $1.33^{\mathrm{pq}}$ & $2.53^{1}$ & $3.80^{\mathrm{Ij} \mathrm{klm}}$ & $1.00^{\mathrm{h}}$ \\
\hline s11 & $0.57^{\mathrm{fgh}}$ & $1.07^{\mathrm{klmn}}$ & $2.00^{\text {ghij }}$ & $3.50^{\text {bcdefg }}$ & $4.47^{\mathrm{Ab}}$ & $1.00^{\mathrm{h}}$ \\
\hline s23 & $0.70^{\text {cdef }}$ & $1.13^{\mathrm{jklm}}$ & $1.77^{\mathrm{klmn}}$ & $3.50^{\text {bcdefg }}$ & $4.47^{\mathrm{Ab}}$ & $0.67^{\mathrm{h}}$ \\
\hline s34 & $0.77^{\mathrm{bcd}}$ & $1.07^{\mathrm{klmn}}$ & $1.78^{\mathrm{jklmn}}$ & $3.27^{\text {efghij }}$ & $4.03^{\text {Efghi }}$ & $0.67^{\mathrm{h}}$ \\
\hline s39 & $0.77^{\mathrm{bcd}}$ & $1.07^{\mathrm{klmn}}$ & $1.67^{\mathrm{lmno}}$ & $1.87^{\mathrm{m}}$ & $4.03^{\text {Efghi }}$ & $0.66^{\mathrm{h}}$ \\
\hline $\mathrm{s} 42$ & $0.77^{\mathrm{bcd}}$ & $1.07^{\mathrm{klmn}}$ & $1.67^{\mathrm{Imno}}$ & $1.87^{\mathrm{m}}$ & $4.03^{\text {Efghi }}$ & $0.66^{\mathrm{h}}$ \\
\hline s37 & $0.77^{\mathrm{b}-\mathrm{d}}$ & $1.13^{\mathrm{jklm}}$ & $1.63^{\mathrm{mno}}$ & $2.97^{\mathrm{jk}}$ & $3.70^{\mathrm{Klm}}$ & $0.65^{\mathrm{hi}}$ \\
\hline s46 & $0.70^{\mathrm{c}-\mathrm{f}}$ & $1.58^{\mathrm{de}}$ & $2.67^{\mathrm{b}}$ & $3.55^{\text {bcdef }}$ & $4.22^{\text {Bcdef }}$ & $0.65^{\mathrm{hi}}$ \\
\hline LSD (0.05) & 0.16 & 0.17 & 0.23 & 0.34 & 0.25 & 1.673 \\
\hline$P$-value & $<0.001$ & $<0.001$ & $<0.001$ & $<0.001$ & $<0.001$ & $<0.001$ \\
\hline
\end{tabular}

* Data are means of three replicates. Means on the same column followed by similar letter(s) are not significantly different at $P \geq 0.05$ according to Fisher's protected LSD test. 
TABLE 3: The mean width and length of spores in micron $(\mu \mathrm{m})$ produced by 10 -day-old Colletotrichum gloeosporioides isolates.

\begin{tabular}{|c|c|c|}
\hline Isolate & Width & Length \\
\hline s1 & $5.0^{* \mathrm{a}}$ & $18.2^{\mathrm{a}}$ \\
\hline s 40 & $5.0^{\mathrm{a}}$ & $18.0^{\mathrm{a}}$ \\
\hline s 44 & $5.0^{\mathrm{a}}$ & $18.0^{\mathrm{a}}$ \\
\hline s3 & $4.7^{\mathrm{a}}$ & $17.1^{\mathrm{ab}}$ \\
\hline s5 & $4.7^{\mathrm{a}}$ & $17.1^{\mathrm{ab}}$ \\
\hline s6 & $4.7^{\mathrm{a}}$ & $16.2^{\mathrm{bc}}$ \\
\hline s19 & $3.7^{\mathrm{b}}$ & $16.1^{b c}$ \\
\hline$s 21$ & $3.7^{\mathrm{b}}$ & $16.1^{b c}$ \\
\hline s10 & $3.6^{\mathrm{bc}}$ & $16.0^{\mathrm{bc}}$ \\
\hline s18 & $3.6^{\mathrm{bc}}$ & $15.4^{\mathrm{cd}}$ \\
\hline s25 & $3.6^{\mathrm{bc}}$ & $15.3^{\mathrm{cd}}$ \\
\hline s 28 & $3.6^{b c}$ & $15.2^{\text {cde }}$ \\
\hline s14 & $3.5^{\mathrm{bcd}}$ & $15.2^{\text {cde }}$ \\
\hline s17 & $3.5^{\mathrm{bcd}}$ & $15.2^{\text {cde }}$ \\
\hline s2 & $3.5^{\mathrm{bcd}}$ & $15.1^{\text {cde }}$ \\
\hline$s 22$ & $3.5^{\mathrm{bcd}}$ & $15.0^{\text {cde }}$ \\
\hline s 24 & $3.5^{\mathrm{bcd}}$ & $15.0^{\text {cde }}$ \\
\hline$s 29$ & $3.5^{\mathrm{bcd}}$ & $15.0^{\text {cde }}$ \\
\hline s30 & $3.5^{\mathrm{bcd}}$ & $15.0^{\text {cde }}$ \\
\hline$s 43$ & $3.5^{\mathrm{bcd}}$ & $14.5^{\mathrm{def}}$ \\
\hline s13 & $3.4^{\text {bcde }}$ & $14.4^{\operatorname{defg}}$ \\
\hline s15 & $3.4^{\text {bcde }}$ & $14.2^{\text {defgh }}$ \\
\hline s4 & $3.4^{\text {bcde }}$ & $14.0^{\mathrm{efgh}}$ \\
\hline s8 & $3.4^{\text {bcde }}$ & $14.0^{\mathrm{efgh}}$ \\
\hline s38 & $3.3^{\text {bcde }}$ & $13.5^{\mathrm{fgh}}$ \\
\hline s16 & $3.3^{\text {bcde }}$ & $13.2^{\text {ghi }}$ \\
\hline s 26 & $3.3^{\text {bcde }}$ & $13.2^{\text {ghi }}$ \\
\hline s45 & $3.3^{\text {bcde }}$ & $13.2^{\text {ghi }}$ \\
\hline s9 & $3.3^{\text {bcde }}$ & $13.1^{\mathrm{hi}}$ \\
\hline s12 & $3.2^{\text {cde }}$ & $13.1^{\mathrm{hi}}$ \\
\hline s 20 & $3.2^{\text {cde }}$ & $13.0^{\mathrm{hi}}$ \\
\hline s27 & $3.2^{\text {cde }}$ & $12.2^{\mathrm{ij}}$ \\
\hline s31 & $3.2^{\text {cde }}$ & $12.2^{\mathrm{ij}}$ \\
\hline s32 & $3.2^{\text {cde }}$ & $12.2^{\mathrm{ij}}$ \\
\hline s33 & $3.2^{\text {cde }}$ & $12.1^{\mathrm{ij}}$ \\
\hline s35 & $3.2^{\text {cde }}$ & $12.1^{\mathrm{ij}}$ \\
\hline s36 & $3.2^{\text {cde }}$ & $12.1^{\mathrm{ij}}$ \\
\hline s41 & $3.2^{\text {cde }}$ & $12.1^{\mathrm{ij}}$ \\
\hline s7 & $3.2^{\text {cde }}$ & $12.0^{\mathrm{ij}}$ \\
\hline s11 & $3.1^{\mathrm{de}}$ & $12.0^{\mathrm{ij}}$ \\
\hline$s 23$ & $3.1^{\mathrm{de}}$ & $11.1^{\mathrm{jk}}$ \\
\hline s34 & $3.1^{\text {de }}$ & $11.0^{\mathrm{jk}}$ \\
\hline s39 & $3.1^{\mathrm{de}}$ & $11.0^{\mathrm{jk}}$ \\
\hline $\mathrm{s} 42$ & $3.1^{\mathrm{de}}$ & $10.4^{\mathrm{k}}$ \\
\hline s37 & $3.0^{\mathrm{e}}$ & $10.3^{\mathrm{k}}$ \\
\hline s46 & $3.0^{\mathrm{e}}$ & $10.3^{\mathrm{k}}$ \\
\hline LSD & 0.4031 & 1.2096 \\
\hline$P$ value & $<0.001$ & $<0.001$ \\
\hline
\end{tabular}

${ }^{*}$ Data are means of three replicates. Means on the same column followed by similar letter(s) are not significantly different at $P \geq 0.05$ according to Fisher's protected LSD test.

characteristic of C. gloeosporioides in vitro. The cultures produced spores which were straight with rounded end, ranging within $3.0-5.0 \mu \mathrm{m}$ in width and $10.3-18.2 \mu \mathrm{m}$ in
TABle 4: Colletotrichum and Pestalotiopsis isolates with their Genbank accession number.

\begin{tabular}{|c|c|c|c|}
\hline Species & Culture & Location & Genbank number \\
\hline \multirow{12}{*}{$\begin{array}{l}\text { Colletotrichum } \\
\text { gloeosporioides }\end{array}$} & $14^{\mathrm{a}}$ & \multirow{12}{*}{$\begin{array}{l}\text { Murang'a } \\
\text { County }\end{array}$} & MG013524 \\
\hline & $39^{\mathrm{a}}$ & & MG013525 \\
\hline & $4^{\mathrm{b}}$ & & MG013527 \\
\hline & $5^{\mathrm{b}}$ & & MG013528 \\
\hline & $6^{\mathrm{b}}$ & & MG013529 \\
\hline & $8^{\mathrm{b}}$ & & MG013530 \\
\hline & $10^{\mathrm{b}}$ & & MG013531 \\
\hline & $11^{\mathrm{b}}$ & & MG013532 \\
\hline & $12^{\mathrm{b}}$ & & MG013533 \\
\hline & $13^{\mathrm{b}}$ & & MG013534 \\
\hline & $9^{\mathrm{b}}$ & & MG013535 \\
\hline & $7^{\mathrm{b}}$ & & MG013536 \\
\hline $\begin{array}{l}\text { Colletotrichum } \\
\text { boninense }\end{array}$ & $26^{\mathrm{a}}$ & $\begin{array}{l}\text { Murang'a } \\
\text { County }\end{array}$ & MG013526 \\
\hline \multirow{10}{*}{$\begin{array}{l}\text { Pestalotiopsis } \\
\text { microspora }\end{array}$} & $27^{\mathrm{a}}$ & \multirow{10}{*}{$\begin{array}{c}\text { Murang'a } \\
\text { County }\end{array}$} & MG013537 \\
\hline & $30^{\mathrm{a}}$ & & MG013538 \\
\hline & $6^{\mathrm{a}}$ & & MG013539 \\
\hline & $28^{\mathrm{a}}$ & & MG013540 \\
\hline & $2^{\mathrm{a}}$ & & MG013541 \\
\hline & $31^{\mathrm{a}}$ & & MG013542 \\
\hline & $32^{\mathrm{a}}$ & & MG013543 \\
\hline & $4^{\mathrm{a}}$ & & MG013544 \\
\hline & $25^{\mathrm{a}}$ & & MG013545 \\
\hline & $18^{\mathrm{a}}$ & & MG013546 \\
\hline
\end{tabular}

length, characteristic of Colletotrichum gloeosporioides as also reported by Chowdappa et al. [32].

The remaining 34 isolates had whitish cream mycelium with black fruiting structure, acervuli on the upper side, and had light orange to orange at lower side. These isolates produced spores having 3-4 septa and 2 or 3 appendages, characteristics of Pestalotiopsis microspora as reported by Elargawy [33]. Further molecular characteristic of these isolates using universal primers ITS1 and ITS4 confirmed the species as Pestalotiopsis microspora as discussed below in molecular characterisation section.

The differences observed in cultural and morphological characters of the isolates could be associated with their genetic variations and repeated subculturing [34, 35]. Further, the isolates had significantly different growth rate at $P<0.05$ (Table 2). Such growth rate among C. gloeosporioides isolates was also reported by Zakaria and Bailey [31]. Overall the proportion in percentage (\%) of the variance in mycelia radial diameter that was predictable from days for the isolates varied from the lowest $(R 2=0.812)$ to highest value $(R 2=$ 0.993).

In this study, sporulation by Colletotrichum gloeosporioides isolates exhibited a wide range of mean number of spores per isolate from lowest $0.67 \times 10^{6}$ to the highest $9.00 \times$ $10^{6}$ spores per $\mathrm{ml}$ (Table 3 ) similar to observation made by 
Peres et al. [9]. These mean numbers of spores per ml differed significantly at $P<0.05$ among isolates (Table 3 ).

The spores observed in this study were cylindrical and straight with smooth round end. Similar spores of $C$. gloeosporioides were observed by Chowdappa et al. [32]. The spore size varied significantly at $P<0.05$ among isolates ranging within $3.0-5.0 \mu \mathrm{m}$ in width and $10.3-18.2 \mu \mathrm{m}$ in length (Table 3 ). Overall, spore size in terms of width and length differed significantly at $P<0.05$ among isolates.

\subsection{Phylogenetic Studies of Colletotrichum gloeosporioides} and Pestalotiopsis microspora. Phylogenetic results showed that ribosomal internal transcribed spacers (ITS) DNA can be used to indicate the relationships within Colletotrichum and Pestalotiopsis species. The study identified Colletotrichum gloeosporioides and Colletotrichum boninense based on a randomly selected sample of 13 sequences of Colletotrichum gloeosporioides isolates using ITS4 (universal) and CgInt (C. gloeosporioides specific) primers yielding single band of $450 \mathrm{bp}$ and $98-100 \%$ homology with nucleotide sequence of ITS region of DNA with C. gloeosporioides isolates in the Genbank (Figure 1). This was in agreement with the nucleotide sequence of ITS region of ribosomal DNA of Colletotrichum gloeosporioides isolates from orchids amplified using specific primers CgInt and ITS4 as reported by Chowdappa et al. [32]. Further, molecular identification of Pestalotiopsis microspora was based on 10 randomly selected sequences of Pestalotiopsis microspora isolates using universal ITS1 and ITS4 primers for DNA sequencing.

Twelve isolates of C. gloeosporioides, one isolate of $C$. boninense, and ten isolates of $P$. microspora from the study area gave identical sequences of the published sequences in the Genbank for the same species with 94\%, 98\%, and $100 \%$ bootstraps value, respectively (Figure 1). Colletotrichum gloeosporioides identified in this study has been reported as the most common and wide spread pathogen in all avocado growing region worldwide [36-38]. Further it has been associated with infection of other hosts such as almond, coffee, guava, apple, dragon fruit, cassava, mango, sorghum, and strawberry $[2,15,39]$. Though $C$. boninense identified is not very common in the study area, its sequence was identical to published sequence (KX 343044.1 and KU356916.1) in the Genbank. This species, among others like Colletotrichum acutatum, Colletotrichum godetiae, C. fioriniae, C. aenigma, and Colletotrichum gigasporum, has been reported to cause anthracnose of avocado [11, 16, 40, 41].

The ten randomly sampled isolates of $P$. microspora identified in this study showed $100 \%$ identity with the published sequences in the Genbank thereby confirming their identity (Figure 1). Pestalotiopsis species are widespread in both tropical and temperate region [42, 43]. Individual species of Pestalotiopsis are known to cause infection on a wide range of hosts $[44,45]$. Pestalotiopsis clavispora is known to cause stem end rot of avocado [6]; P. versicolor has been reported as a causal agent of anthracnose in avocado [46]. $P$. palmarum known to cause leaf spot and fruit canker in avocado [47]. Though $P$. microspora is prevalent in both tropics and subtropics, its association with host plants is not well researched [48]. It has been regarded both as an endophyte and as a pathogen causing postharvest diseases [49]. It has been reported to cause scab disease of guava fruits in Hawaii [48]. In this study, the fungus was isolated from diseased avocado fruits showing symptoms associated with anthracnose disease of avocado. However, despite its prevalence, $P$. microspora and its role in plant ecology are poorly understood [49].

Conclusively, Colletotrichum gloeosporioides, Colletotrichum boninense, and Pestalotiopsis microspora were identified for the first time as the causal agents of anthracnose of avocado in Kenya through cultural, morphological, and molecular techniques.

\section{Conflicts of Interest}

The authors declare that there are no conflicts of interest regarding the publication of this article.

\section{Authors' Contributions}

S. K. Kimaru conceived, designed, and performed experiments and was responsible for collection of diseased avocado fruits and isolation of fungi, morphological identification of the fungi, DNA extraction, running gel electrophoresis, analysis of sequence and construction of phylogenetic tree, data analysis, and drafting manuscript. R. C. Cheruiyot participated in the creation of the concept of the manuscript, revision of the manuscript, and approval of the manuscript. J. Mbaka was responsible for confirmation of the identity of fungi morphologically, methodical consultation in revision of the manuscript, and approval of manuscript. A. Alakonya was responsible for revision of the manuscript mainly regarding molecular section and approval of manuscript. E. Monda participated in the conception and design of the experiment, identification of fungi morphologically, sequence analysis, and methodical consultation in verification and approval of the manuscript.

\section{Acknowledgments}

The authors acknowledge Kenyatta University for granting study leave, Kenya Agricultural Research and Livestock Organisation (KARLO), Kandara and Kenya Plant Health Inspectorate Services (KEPHIS) where morphological and molecular studies were conducted, respectively, and Inqaba Biotec, South Africa, for DNA sequencing.

\section{References}

[1] A. T. Djami-Tchatchou, C. J. Straker, and F. Allie, “454 Sequencing for the identification of genes differentially expressed in avocado fruit (cv. Fuerte) infected by Colletotrichum gloeosporioides," Journal of Phytopathology, vol. 160, no. 9, pp. 449-460, 2012.

[2] J. E. Erpelding, "Field assessment of anthracnose disease response for the Sorghum germplasm collection from the Mopti region," American Journal of Agricultural and Biological Sciences, vol. 5, no. 3, pp. 363-369, 2010.

[3] C. A. Onyeani and N. A. Amusa, "Incidence and severity of anthracnose in mango fruits and its control with plant extracts 
in south west nigeria," International Journal of Agricultural Research, vol. 10, no. 1, pp. 33-43, 2015.

[4] M. Sharma and S. Kulshrestha, "Colletotrichum gloeosporioides: an anthracnose causing pathogen of fruits and vegetables," Biosciences, Biotechnology Research Asia, vol. 12, no. 2, pp. 1233-1246, 2015.

[5] H. V. Silva-Rojas and G. D. Ávila-Quezada, "Phylogenetic and morphological identification of Colletotrichum boninense: a novel causal agent of anthracnose in avocado," Plant Pathology, vol. 60, no. 5, pp. 899-908, 2011.

[6] A. Valencia, R. Torres, and B. Latorre, "First report of Pestalotiopsis clavispora and Pestalotiopsis spp. causing postharvest stem end rot of avocado in Chile," Plant Disease, vol. 95, no. 4, pp. 492-493, 2011.

[7] H. J. Boesewinkel, "A list of 142 new plant disease recordings from New Zealand and short notes on three diseases," Australasian Plant Pathology, vol. 11, no. 4, pp. 40-43, 1982.

[8] F. Liu, M. Wang, U. Damm, P. W. Crous, and L. Cai, "Species boundaries in plant pathogenic fungi: a Colletotrichum case study," BMC Evolutionary Biology, vol. 16, no. 1, article 649, 2016.

[9] N. A. R. Peres, E. E. Kuramae, M. S. C. Dias, and N. L. De Souza, "Identification and characterization of Colletotrichum spp. affecting fruit after harvest in Brazil," Journal of Phytopathology, vol. 150, no. 3, pp. 128-134, 2002.

[10] D. G. Rodriguez-Sanchez, M. Flores-García, C. Silva-Platas et al., "Isolation and chemical identification of lipid derivatives from avocado (Persea americana) pulp with antiplatelet and antithrombotic activities," Food \& Function, vol. 6, no. 1, pp. 193203, 2015.

[11] M. V. Valle and A. Campos-Martínez, "First report of avocado anthracnose caused by Colletotrichum karstii in Mexico," Plant Disease, vol. 100, no. 2, p. 534, 2016.

[12] S. L. Willingham, A. W. Cooke, L. M. Coates, and K. G. Pegg, "Pepper spot: a new preharvest Colletotrichum disease of avocado cv. Hass," Australasian Plant Pathology, vol. 29, no. 2, p. $151,2000$.

[13] D. F. Farr, M. C. Aime, A. Y. Rossman, and M. E. Palm, "Species of colletotrichum on agavaceae," Mycological Research, vol. 110, no. 12, pp. 1395-1408, 2006.

[14] B. Schaffer, B. Wolstenholme, and A. Whiley, The Avocado: Botany, Production and Uses, CABI, 2013.

[15] G. N. Agrios, Plant Pathology, Elsevier Academic Press, 2005.

[16] E. Dann, R. Ploetz, L. Coates, and K. Pegg, "Foliar, fruit and soilborne diseases," in The Avocado: Botany, Production and Uses, pp. 380-422, 2013.

[17] A. Gautam, "Colletotrichum gloeosporioides: biology, pathogenicity and management in India," Plant Physiology \& Pathology, vol. 2, no. 2, article 2, 2014.

[18] K. Pernezny and R. Marlatt, "Diseases of avocado in Florida," Plant Pathol Fact Sheet 21, 2000.

[19] L. Wasilwa, J. Njuguna, and E. Okoko, Status of Avocado Production in Kenya, Kenya Agricultural Research Institute, Nairobi, Kenya, 2004.

[20] Y. Choi, K. Hyde, and W. Ho, Fungal Diversity, Fungal Diversity Press, 1999.

[21] S. Freeman, T. Katan, and E. Shabi, "Characterization of Colletotrichum species responsible for anthracnose diseases of various fruits," Plant Disease, vol. 82, no. 6, pp. 596-605, 1998.

[22] A. Nagamani and I. K. Kunwar, Handbook of Soil Fungi, Edited by K. Indra and C. Manoharachary, I.K. International, 2009.
[23] B. Liu, F. J. Louws, T. B. Sutton, and J. C. Correll, "A rapid qualitative molecular method for the identification of Colletotrichum acutatum and C. gloeosporioides," European Journal of Plant Pathology, vol. 132, no. 4, pp. 593-607, 2012.

[24] S. Kumar, G. Stecher, and K. Tamura, "MEGA7: Molecular Evolutionary Genetics Analysis version 7.0 for bigger datasets," Molecular Biology and Evolution, vol. 33, no. 7, pp. 1870-1874, 2016.

[25] K. Takahashi and M. Nei, "Efficiencies of fast algorithms of phylogenetic inference under the criteria of maximum parsimony, minimum evolution, and maximum likelihood when a large number of sequences are used," Molecular Biology and Evolution, vol. 17, no. 8, pp. 1251-1258, 2000.

[26] H. Kishino and M. Hasegawa, "Evaluation of the maximum likelihood estimate of the evolutionary tree topologies from DNA sequence data, and the branching order in hominoidea," Journal of Molecular Evolution, vol. 29, no. 2, pp. 170-179, 1989.

[27] K. Domsch, W. Gams, and T. Anderson, Compendium of Soil Fungi, vol. 1, Academic Press, 1980.

[28] A. J. Rabha, A. Naglot, G. D. Sharma et al., "Morphological and molecular diversity of endophytic Colletotrichum gloeosporioides from tea plant, Camellia sinensis (L.) O. Kuntze of Assam, India," Journal of Genetic Engineering and Biotechnology, vol. 14, no. 1, pp. 181-187, 2016.

[29] C. F. B. Silva and S. J. Michereff, "Biology of Colletotrichum spp. and epidemiology of the anthracnose in tropical fruit trees," Revista Caatinga, vol. 26, no. 4, pp. 130-138, 2014.

[30] K. D. Hyde, R. H. Nilsson, S. A. Alias et al., "One stop shop: backbones trees for important phytopathogenic genera: I (2014)," Fungal Diversity, vol. 67, no. 1, pp. 21-125, 2014.

[31] M. Zakaria and J. A. Bailey, "Morphology and cultural variation among Colletotrichum isolates obtained from tropical forest nurseries," Journal of Tropical Forest Science, vol. 12, no. 1, pp. $1-20,2000$.

[32] P. Chowdappa, C. S. Chethana, R. Bharghavi, H. Sandhya, and R. P. Pant, "Morphological and molecular characterization of Colletotrichum gloeosporioides (Penz) Sac. isolates causing anthracnose of orchids in India," Biotechnology, Bioinformatics and Bioengineering, vol. 2, no. 1, pp. 567-572, 2012.

[33] E. El-Argawy, "haracterization and control of Pestalotiopsis spp. the causal fungus of guava scabby canker in el-beheira governorate, Egypt," International Journal of Phytopathology, vol. 4, no. 3, pp. 121-136, 2016.

[34] A. Vidyalakshmi and C. V. Divya, "New report of Colletotrichum gloeosporioides causing anthracnose of Pisonia alba in India," Archives of Phytopathology and Plant Protection, vol. 46, no. 2, pp. 201-204, 2013.

[35] W. V. Parker, P. R. Johnston, and U. Damm, "Colletotrichum gloeosporioides species complex," Studies in Mycology, vol. 73, pp. 115-180, 2012.

[36] J. O. Honger, S. K. Offei, K. A. Oduro, G. T. Odamtten, and S. T. Nyaku, "Identification and molecular characterisation of Colletotrichum species from avocado, citrus and pawpaw in Ghana," South African Journal of Plant and Soil, vol. 33, no. 3, pp. 177-185, 2016.

[37] Y. Siddiqui and A. Ali, "Chapter 11-Colletotrichum gloeosporioides (Anthracnose)," in Postharvest Decay, 2014.

[38] M. Twizeyimana, H. Förster, V. McDonald, D. H. Wang, J. E. Adaskaveg, and A. Eskalen, "Identification and pathogenicity of fungal pathogens associated with stem-end rot of avocado in California," Plant Disease, vol. 97, no. 12, pp. 1580-1584, 2013. 
[39] R. Dean, J. A. L. Van Kan, Z. A. Pretorius et al., "The Top 10 fungal pathogens in molecular plant pathology," Molecular Plant Pathology, vol. 13, no. 4, pp. 414-430, 2012.

[40] F. R. Giblin, L. M. Coates, and J. A. G. Irwin, "Pathogenic diversity of avocado and mango isolates of Colletotrichum gloeosporioides causing anthracnose and pepper spot in Australia," Australasian Plant Pathology, vol. 39, no. 1, pp. 50-62, 2010.

[41] A. Hernandez-Lauzardo and A. Campos-Martínez, "First report of Colletotrichum godetiae causing anthracnose on avocado in Mexico," Plant Disease, vol. 99, no. 10, p. 555, 2015.

[42] G. Ding, Z. Zheng, S. Liu, H. Zhang, L. Guo, and Y. Che, "Photinides A-F, cytotoxic benzofuranone-derived $\gamma$-lactones from the plant endophytic fungus Pestalotiopsis photiniae," Journal of Natural Products, vol. 72, no. 5, pp. 942-945, 2009.

[43] L. Liu, S. Liu, X. Chen, L. Guo, and Y. Che, "Pestalofones A-E, bioactive cyclohexanone derivatives from the plant endophytic fungus Pestalotiopsis fici," Bioorganic \& Medicinal Chemistry, vol. 17, no. 2, pp. 606-613, 2009.

[44] Y. Suto and T. Kobayashi, "Taxonomic studies on the species of Pestalotiopsis, parasitic on conifers in Japan," Transactions of the Mycological Society of Japan, 1993.

[45] A. M. Ismail, G. Cirvilleri, and G. Polizzi, "Characterisation and pathogenicity of Pestalotiopsis uvicola and Pestalotiopsis clavispora causing grey leaf spot of mango (Mangifera indica L.) in Italy," European Journal of Plant Pathology, vol. 135, no. 4, pp. 619-625, 2013.

[46] J. Darvas, "Fungi associated with pre-and postharvest diseases of avocado fruit at Westfalia Estate, South Africa," Phytophylactica, vol. 19, no. 1, pp. 83-86, 1987.

[47] M. Kamhawy, M. Hassan, and S. Sharkawy, "Morphological and phylogenetic characterization of pestalotiopsis in relation to host association," Egyptian Journal of Agricultural Research, vol. 89, no. 1, pp. 1-16, 2011.

[48] L. M. Keith, M. E. Velasquez, and F. T. Zee, "Identification and characterization of Pestalotiopsis spp. causing scab disease of guava, Psidium guajava, in Hawaii," Plant Disease, vol. 90, no. 1, pp. 16-23, 2006.

[49] A. M. Metz, A. Haddad, J. Worapong et al., "Induction of the sexual stage of Pestalotiopsis microspora, a taxol-producing fungus," Microbiology, vol. 146, no. 8, pp. 2079-2089, 2000. 


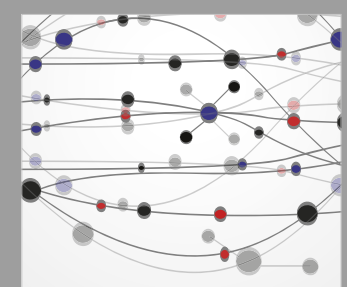

The Scientific World Journal
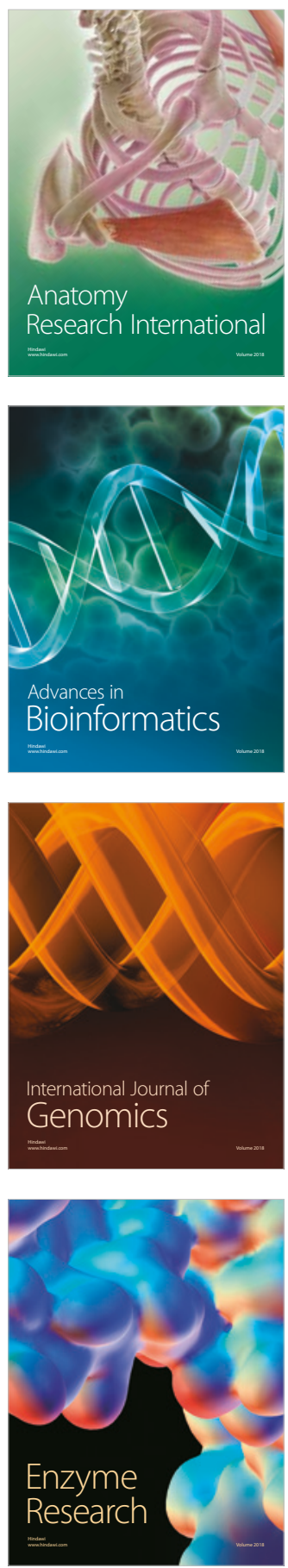
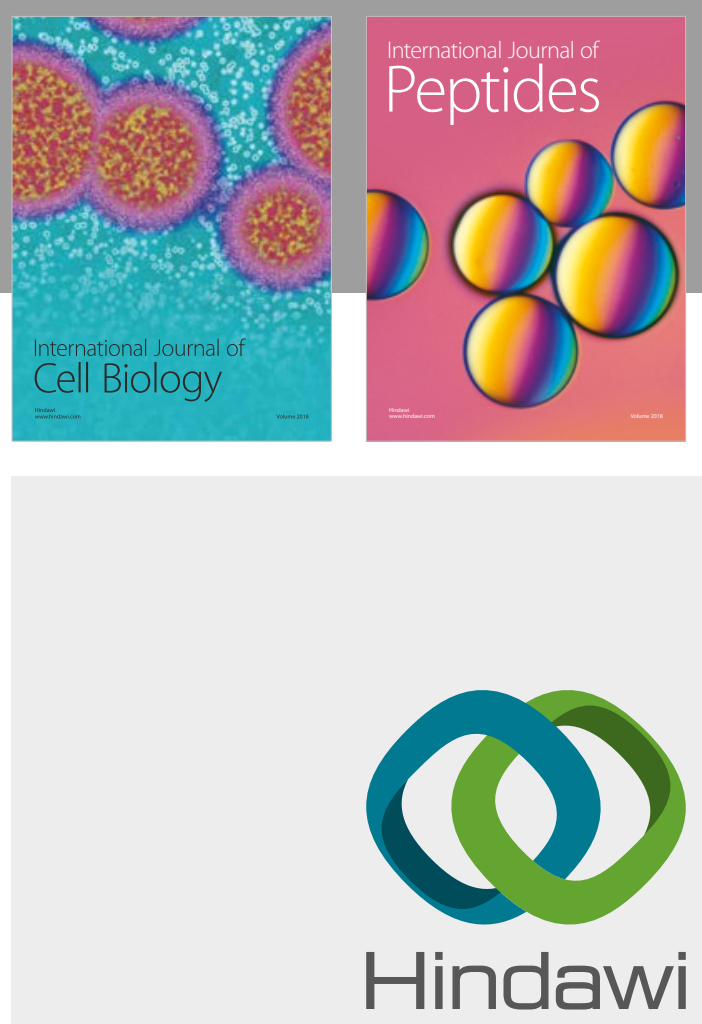

Submit your manuscripts at

www.hindawi.com
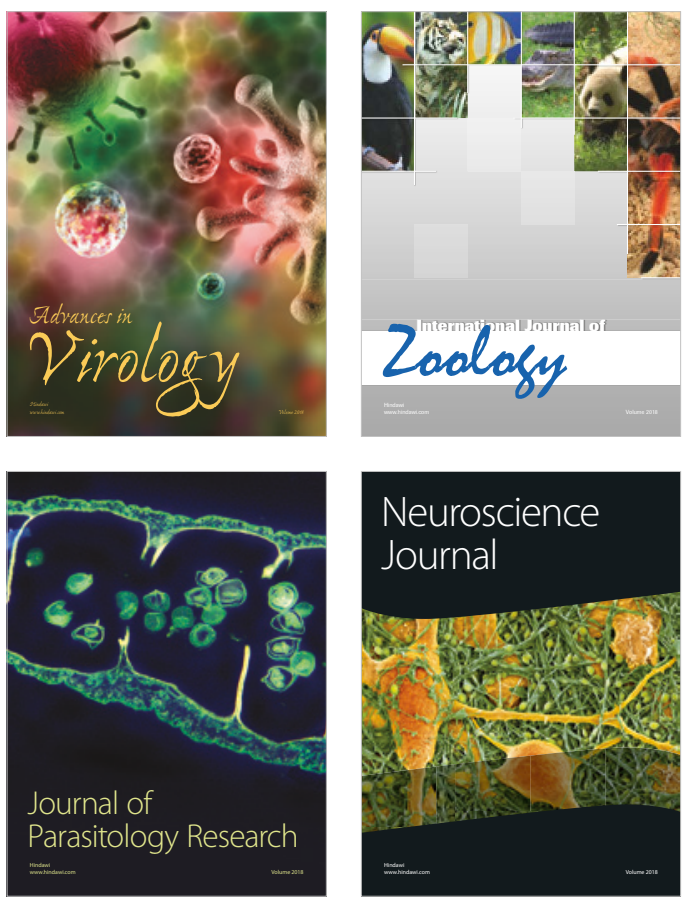
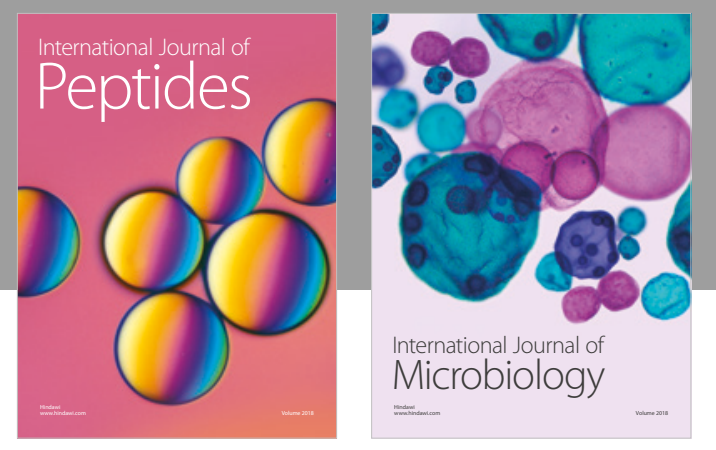

nternational Journal of Microbiology
Journal of
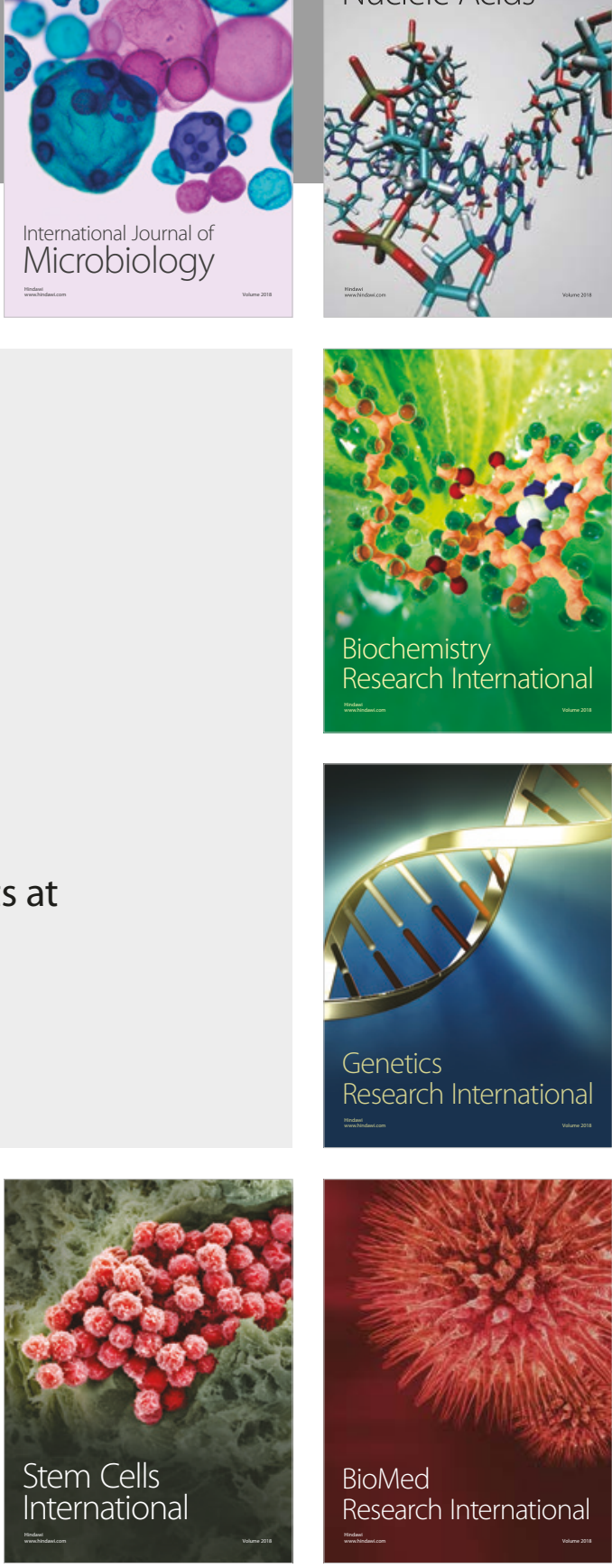
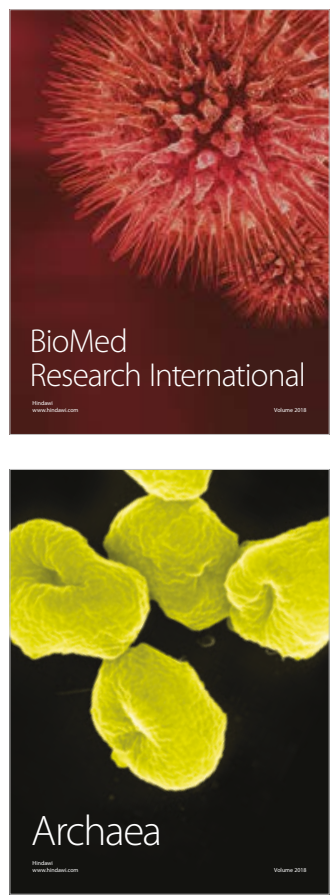